\title{
Logarithmic moments of relaxation time distributions
}

\author{
Reiner Zorna) \\ Forschungszentrum Jülich, IFF, D-52425 Jülich, Germany
}

(Received 22 October 2001; accepted 3 December 2001)

\begin{abstract}
In this paper a novel way to quantify "nonexponential" relaxations is described. So far, this has been done in two ways: (1) by fitting empirical functions with a small number of parameters, (2) by calculation of the underlying distribution function $g(\ln \tau)$ of (exponential) relaxations using regularization methods. The method described here is intermediate, it does not assume a specific functional form but also does not aim at the complete distribution $g(\ln \tau)$ but only its logarithmic moments $\left\langle(\ln \tau)^{k}\right\rangle$. It is shown that these exist (in contrast to the linear moments) and can be calculated analytically for all currently used empirical descriptions of nonexponential relaxations. Therefore, the logarithmic moments represent a common basis for comparing literature data from authors which prefer different empirical formulas (e.g., those of Kohlrausch and Havriliak-Negami). The logarithmic moments are also shown to be related in a simple way to the (linear) moments of an underlying distribution of activation energies giving them a physical significance. (C) 2002 American Institute of Physics. [DOI: 10.1063/1.1446035]
\end{abstract}

\section{INTRODUCTION}

It is well known-especially from glass-forming systems - that the relaxation of certain quantities in response to a step function often does not follow a simple exponential law. This is found in dielectric, mechanical, light scattering, neutron scattering, and many other relaxation techniques. The mathematical description of such a behavior has been of considerable interest in the literature. In this paper the results will be formulated in terms of dielectric relaxation but it is simple to translate them into the context of the other methods.

For dielectric relaxation the time-dependent dielectric function $\epsilon(t)$ can be written in a normalized way

$$
\phi(t)=\frac{\epsilon_{0}-\epsilon(t)}{\epsilon_{0}-\epsilon_{\infty}},
$$

where $\phi(t)$, the normalized relaxation function, in the most simple case is $\exp (-t / \tau)$. Then (and only then) one obtains for the complex frequency-dependent dielectric function $\epsilon(\omega)$ Debye's result:

$$
\frac{\epsilon(\omega)-\epsilon_{\infty}}{\epsilon_{0}-\epsilon_{\infty}}=\frac{1}{1+i \omega \tau} .
$$

Because the exponential relaxation or equivalently the Debye description of the susceptibility often fail in the description of experimental data a plethora of empirical functions have been developed. The oldest attempt dating back to Kohlrausch ${ }^{1}$ is to set

$$
\phi(t)=\exp \left(-\left(t / \tau_{K}\right)^{\beta}\right),
$$

i.e., introducing a "stretching" parameter $0<\beta \leqslant 1$ in the logarithmic time. Other authors introduced modifications of the Debye expression to

\footnotetext{
a)Electronic mail: r.zorn@kfa-juelich.de
}

$$
\frac{\epsilon(\omega)-\epsilon_{\infty}}{\epsilon_{0}-\epsilon_{\infty}}=\frac{1}{\left(1+(i \omega \tau)^{\alpha}\right)^{\gamma}}
$$

with $\alpha$ or $\gamma$ or both between zero and one. ${ }^{2-5}$ Another attempt is based on modification of the imaginary part of (2) which is often the measured quantity in dielectric experiments-to

$$
\epsilon^{\prime \prime}(\omega)=\frac{A}{\left(\omega / \omega_{1}\right)^{-a}+\left(\omega / \omega_{2}\right)^{b}},
$$

where $0<a, b \leqslant 1 .^{5-7}$ For $a=b=1, \omega_{1}=\omega_{2}=1 / \tau$, and $A$ $=1$. Eq. (5) reduces to the Debye expression.

The few parameters determining the relaxation function or susceptibility in the preceding descriptions can usually be determined unambiguously by least-squares fit to the experimental data. Nevertheless, it remains questionable whether the parameters obtained in this way have any physical significance. Also there is often no a priori reason to prefer one of the descriptions and the fits are equally well and allow no a posteriori preference too.

A much more general description of experimentally measured relaxation functions is possible by expressing them as a superposition of exponential relaxations

$$
\phi(t)=\int_{-\infty}^{+\infty} d \ln \tau g(\ln \tau) \exp (-t / \tau)
$$

where $g(\ln \tau)$ is a distribution function which is always positive and whose integral is normalized to one. From probability theory it is known that it is possible to obtain a distribution $g(\ln \tau)$ from $\phi(t)$ according to Eq. (6) if and only if $\phi(t)$ is completely monotone. ${ }^{8}$ This means that the derivatives $\phi^{(n)}(t)=d^{n} \phi / d t^{n}$ must have alternating signs over the whole range $t=0, \ldots, \infty$ : $(-1)^{n} \phi^{(n)}(t) \geqslant 0$. In practice it turns out to be a rather weak condition which is fulfilled for nearly all experimentally obtained relaxation functions. 
Equivalently, the susceptibility can then be written as a superposition of Debye expressions

$$
\frac{\epsilon(\omega)-\epsilon_{\infty}}{\epsilon_{0}-\epsilon_{\infty}}=\int_{-\infty}^{+\infty} d \ln \tau g(\ln \tau) \frac{1}{1+i \omega \tau} .
$$

The inversion of Eq. (6) from experimental $\phi(t)$ data or (7) from measured $\epsilon^{\prime \prime}(\omega)$ or $\epsilon^{\prime}(\omega)$ is a difficult task. It is a so-called "ill-defined" problem which can numerically only be solved by regularization methods and not by least-squares algorithms. While these methods have been used in light scattering for quite some time (CONTIN algorithm ${ }^{9}$ ) they have been introduced to dielectric spectroscopy only recently. ${ }^{10}$

Of course it is also possible to assume simple distribution functions $g(\ln \tau)$ with a small number of parameters. Then it is again possible to use least-squares methods but arguments concerning the arbitrariness of the choice of the function can be put forward again.

In this paper a description of nonexponential relaxations will be proposed which is intermediate between those described above (that by a small number of exponent parameters and that by a whole distribution function): a description by logarithmic moments of the distribution function.

The choice of this description is motivated by the qualitative criteria often mentioned only verbally without giving a quantitative expression in the literature: the characteristic time of the relaxation, the width (or stretching) of the relaxation, and its asymmetry. The aim of this paper is to define these three properties of a relaxation as numerical quantities which can be obtained for all above-mentioned concepts in a mathematically not too complicated way.

Already the problem of defining the characteristic time makes clear that logarithmic moments are well-suited for this task. Probably most often the inverse of the maximum in the dielectric loss is used as a characteristic time of a non-Debye relaxation. Especially for broad distributions this value can be difficult to obtain and depend strongly on which empirical function is used to describe the data.

Therefore, some authors use the average relaxation time $\langle\tau\rangle$. While this value is well-defined and simply calculable, e.g., for the Kohlrausch expression (3) it is undefined as soon as there is a fractal behavior of the low frequency loss part, e.g., for expression (4) with $\gamma<1$. The average relaxation rate $\left\langle\tau^{-1}\right\rangle$ is-even worse- undefined for nearly all the empirical functions in practical use because they all show a fractal behavior for high frequencies.

A way to avoid these divergences due to the fractal wings of the distribution function is the use of the average logarithmic relaxation time

$$
\langle\ln \tau\rangle=\int_{-\infty}^{+\infty} d \ln \tau \ln \tau
$$

as the characteristic time of a non-Debye relaxation.

Then it is straightforward to define the shape properties of the relaxation time distribution by the higher moments of $\ln \tau$, the relaxation width as the variance of distribution of logarithmic relaxation times

$$
\sigma_{\ln \tau}^{2}=\left\langle(\ln \tau)^{2}\right\rangle-\langle\ln \tau\rangle^{2}
$$

and the asymmetry as the skewness of distribution of logarithmic relaxation times

$$
\gamma_{1 \ln \tau}=\mu_{3 \ln \tau} / \sigma_{\ln \tau}^{3},
$$

where

$$
\mu_{3 \ln \tau}=\left\langle(\ln \tau)^{3}\right\rangle-3\left\langle(\ln \tau)^{2}\right\rangle\langle\ln \tau\rangle+2\langle\ln \tau\rangle^{3}
$$

is the third centralized moment of the distribution of logarithmic relaxation times.

Of course this formalism could be extended to higher moments allowing a more accurate description of the relaxation shape. But the fact that most of the empirical formulas contain only one or two shape parameters indicates that a characterization by three moments is sufficient.

\section{CALCULATION OF LOGARITHMIC MOMENTS}

In the following it is demonstrated that the calculation of the quantities defined by Eqs. (8)-(11) is indeed practicable for any way of defining a nonexponential relaxation.

In the simplest case the definition of a nonexponential relaxation is done directly by the distribution function ${ }^{11-14}$ $g(\ln \tau)$. In this case Eqs. (8)-(11) can be applied directly. Results are shown in the first five rows of Table I.

Somewhat more complicated is the case when the complex dielectric function $\epsilon(\omega)$ is given ${ }^{2-4}$ (rows 6-8 of Table I). In this case the distribution function of relaxation times can be obtained in closed form ${ }^{15}$

$$
\begin{aligned}
g(\ln \tau)= & \frac{1}{2 \pi\left(\epsilon_{0}-\epsilon_{\infty}\right)} \lim _{\omega \rightarrow 0}(\Im(\epsilon(-\omega+i / \tau)) \\
& -\Im(\epsilon(\omega+i / \tau))),
\end{aligned}
$$

and from $g(\ln \tau)$ in turn the logarithmic moments can be calculated. But often the calculation via the loss part $\epsilon^{\prime \prime}(\omega)$ described in the following is easier.

The loss part is obtained from the distribution function as the imaginary part of (7) which can be written as

$$
\frac{\epsilon^{\prime \prime}(\omega)}{\epsilon_{0}-\epsilon_{\infty}}=\int_{-\infty}^{+\infty} d \ln \tau g(\ln \tau) \frac{\operatorname{sech}(\ln \omega+\ln \tau)}{2} .
$$

From this formulation it becomes clear that $\epsilon^{\prime \prime}(\omega)$ is the convolution of $\operatorname{sech}(\ln \omega) / 2$ with the distribution function $g(\ln \tau)$. With this idea it is simple to calculate the moments of $\ln \tau$ from those of $\ln \omega$ using the moment rules for convolution:

$$
\begin{aligned}
& \langle\ln \tau\rangle=-[\ln \omega], \\
& \sigma_{\ln \tau}^{2}=\left[(\ln \omega)^{2}\right]-[\ln \omega]^{2}-\pi^{2} / 4, \\
& \mu_{3 \ln \tau}=-\left[(\ln \omega)^{3}\right]+3\left[(\ln \omega)^{2}\right][\ln \omega]-2[\ln \omega]^{3} .
\end{aligned}
$$

Here the brackets $\left[(\ln \omega)^{n}\right]$ denote the $n$th logarithmic moment of $\omega$ with $\epsilon^{\prime \prime}(\omega)$ taken as a distribution function, namely,

$$
\left[(\ln \omega)^{n}\right]=\frac{2}{\pi\left(\epsilon_{0}-\epsilon_{\infty}\right)} \int_{-\infty}^{+\infty} d \ln \omega \epsilon^{\prime \prime}(\omega)(\ln \omega)^{n} .
$$


TABLE I. Common empirical expressions for nonexponential/non-Debye relaxations and the logarithmic moments of their distribution functions. Notation: $\mathrm{Eu}=0.5772 \ldots$; Euler constant, $\zeta(3)=1.202 \ldots$; Riemann's zeta function, $\psi(x)$ : digamma function; $\psi^{\prime}(x)$ : trigamma function; $\psi^{\prime \prime}(x)$ : tetragamma function

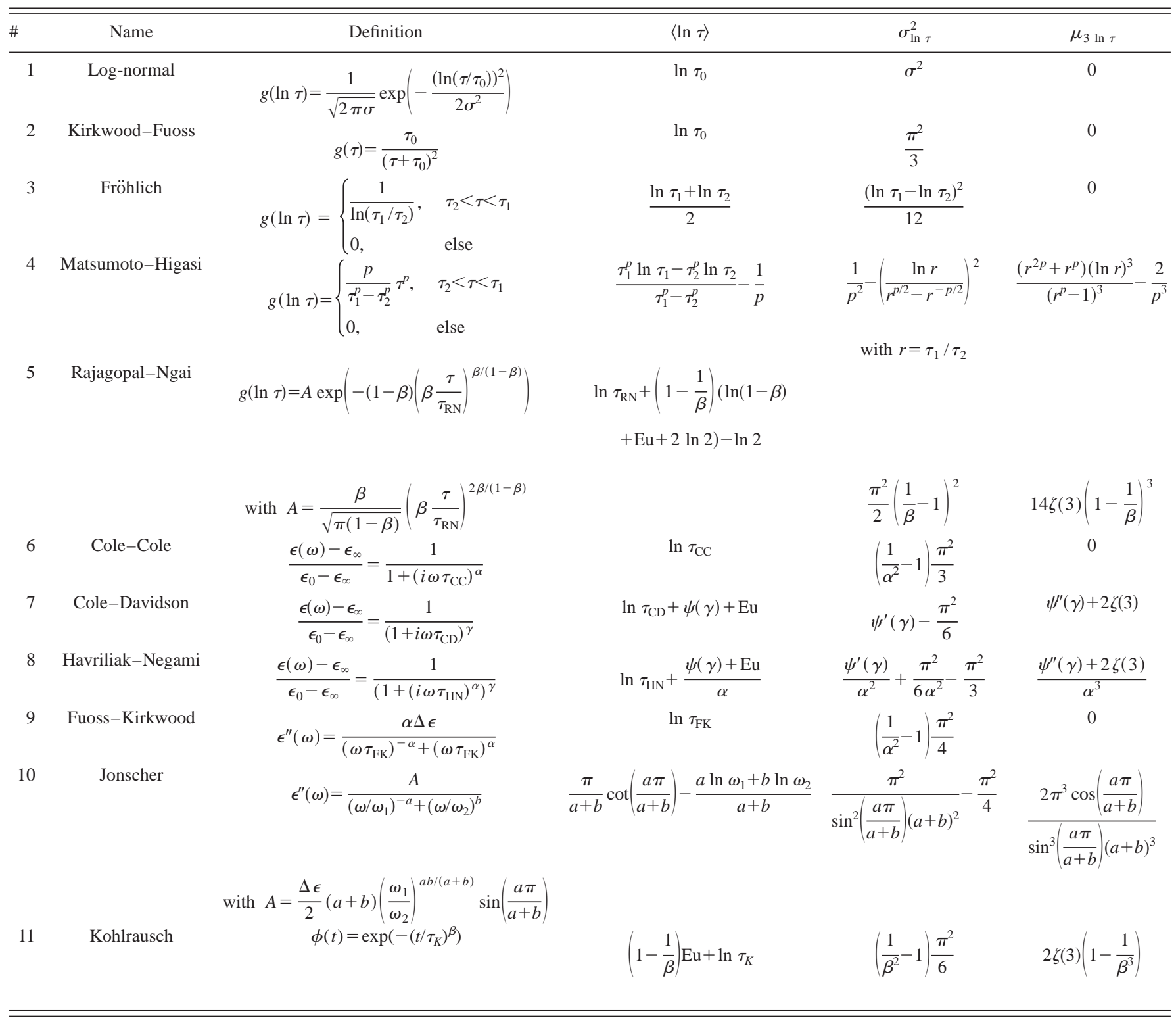

Rows 9 and 10 of Table I show the logarithmic moments calculated in this way for definitions of the dielectric function where only the loss part is given. ${ }^{6,7}$

In a similar way the logarithmic moments can be calculated if the relaxation is defined by a function $\phi(t)$ in time domain. The negative derivative of (6) with respect to $\ln t$ can be written as

$$
\begin{aligned}
-\frac{d \phi}{d \ln t}= & \int_{-\infty}^{+\infty} d \ln \tau g(\ln \tau) \exp (\ln t-\ln \tau \\
& -\exp (\ln t-\ln \tau)) .
\end{aligned}
$$

This is a convolution of $\exp (x-\exp (x))$ with the distribution function $g(\ln \tau)$. The resulting relations for the moments are

$$
\begin{aligned}
& \langle\ln \tau\rangle=\mathrm{Eu}+[\ln t], \\
& \sigma_{\ln \tau}^{2}=\left[(\ln t)^{2}\right]-[\ln t]^{2}-\pi^{2} / 6,
\end{aligned}
$$

$$
\mu_{3 \ln \tau}=\left[(\ln t)^{3}\right]-3\left[(\ln t)^{2}\right][\ln t]+2[\ln t]^{3}+2 \zeta(3)
$$

with the Euler constant $\mathrm{Eu}=0.5772 \ldots$ and $\zeta(3)=1.202 \ldots$, Riemann's zeta function with the argument 3. $\left[(\ln t)^{n}\right]$ is defined for the time coordinate in a similar way as by (17) for frequencies

$$
\left[(\ln t)^{n}\right]=\int_{-\infty}^{+\infty} d \ln t\left(-\frac{d \phi}{d \ln t}\right)(\ln t)^{n} .
$$

Using this method the logarithmic moments of $g(\ln \tau)$ can be calculated for the Kohlrausch function ${ }^{1}$ (row 11 of Table I). For $\beta=1 / 2$ the logarithmic moments are the same as for the Rajagopal-Ngai distribution. This is because the latter is constructed to be the exact distribution of relaxation times of the Kohlrausch function in the special case $\beta=1 / 2$ and a reasonable approximation for other values of $\beta .^{14}$ 


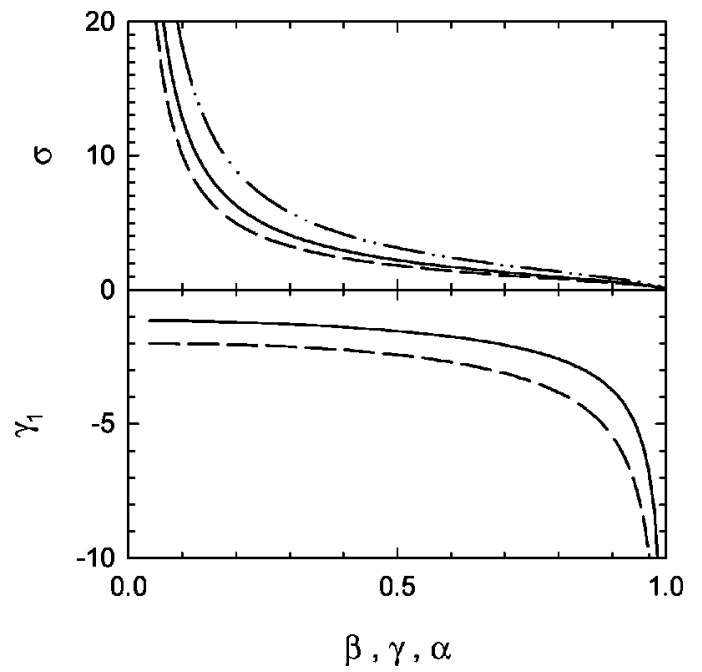

FIG. 1. Width $\sigma_{\ln \tau}$ and skewness $\gamma_{1 \ln \tau}$ of the most common "oneparameter" functions: Kohlrausch (continuous curve), Cole-Davidson (dashed curve), and Cole-Cole (dashed-dotted curve).

For the most commonly used empirical relaxation functions with one adjustable parameter, the Kohlrausch, ColeDavidson, and Cole-Cole expressions, Fig. 1 shows the first and second logarithmic moments. As expected the logarithmic width of the corresponding relaxation time distribution increases with decreasing parameter $\beta, \alpha$, or $\gamma$. If the parameters have the value one all three functions reduce to the Debye/exponential case; thus the width of the distribution vanishes. If the parameters approach zero the width diverges as $\sigma_{\ln \tau} \sim \beta^{-1}, \alpha^{-1}, \gamma^{-1}$. For the asymmetric ones among these functions the absolute value of the skewness increases with increasing parameter $\beta$ or $\gamma$, they even diverge as $\left|\gamma_{1 \ln \tau}\right| \sim(1-\beta)^{-1 / 2},(1-\gamma)^{-1 / 2}$. This is a counterintuitive consequence of normalizing the third moment by the cubed width in definition (10).

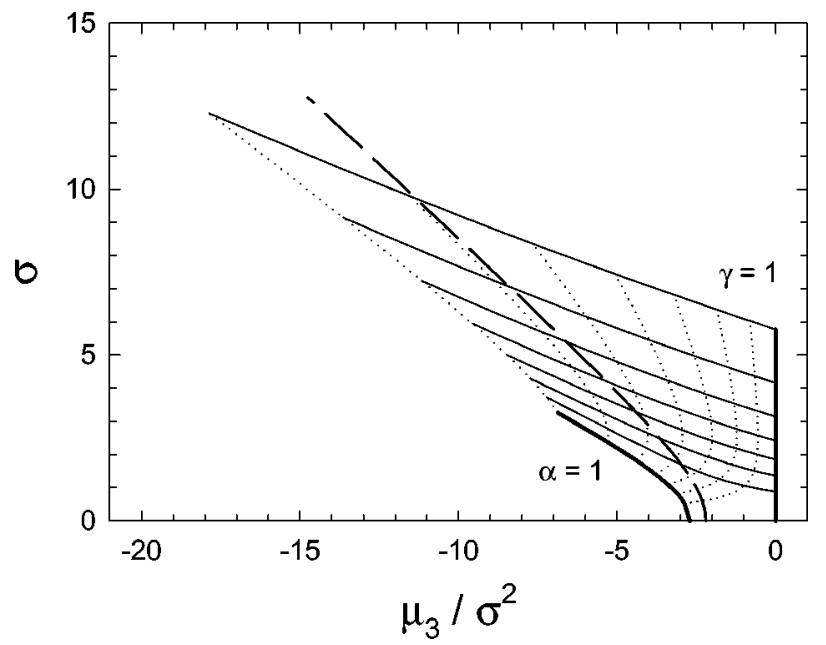

FIG. 2. Width $\sigma_{\ln \tau}$ and (alternatively defined!) skewness $\mu_{3 \ln \tau} / \sigma_{\ln \tau}^{2}$ of the Havriliak-Negami function: The continuous curves represent constant $\alpha=0.3, \ldots, 1$ in steps of 0.1 , the bold curve belongs to the Cole-Davidson limit, $\alpha=1$. The dotted curves represent constant $\gamma=0.3, \ldots, 1$ in steps of 0.1 , the bold curve belongs to the Cole-Cole limit, $\gamma=1$. The dashed curve shows for comparison the same values for the Kohlrausch function with $\beta=0.3, \ldots, 1$.
Figure 2 shows a graphical representation of the results for the two-parameter Havriliak-Negami function. As abscissa an alternative definition of the skewness,

$$
\mu_{3 \ln \tau} / \sigma_{\ln \tau}^{2},
$$

has been chosen to avoid the divergence of definition (10) in the limit $\alpha \rightarrow 1$. It can be seen that the Havriliak-Negami function is able to represent any width of the relaxation but only negative skewness (as has been pointed out by Havriliak and Havriliak ${ }^{16}$ this deficiency can be resolved by allowing $\gamma>1$ with the weaker restriction $\alpha \gamma<1$ but only a few authors actually use this generalization) in a certain range delimited by the Cole-Davidson case labeled by $\alpha=1$ in the plot.

From Fig. 2 and the equivalent plots for the Jonscher and Matsumoto-Higasi functions it seems that the combination "large skewness, small width" cannot be attained. But this is not a mathematical property because distributions with any combination of the first three moments can be constructed. It is rather a general experimental outcome than an a priori limitation that such highly skewed distributions do not occur in reality.

\section{INTERRELATIONS OF EMPIRICAL FUNCTIONS}

It is clear that all the empirical functions listed in Table I are distinct. They only coincide if one is a special case of another, e.g., the Cole-Davidson function and the Havriliak-Negami function. Especially, the Kohlrausch function has no equivalent in the frequency domain. Its Fourier transform is only known for special cases where $\beta$ is a rational number, ${ }^{17}$ e.g., for $\beta=1 / 2$ :

$$
\frac{\epsilon(\omega)-\epsilon_{\infty}}{\epsilon_{0}-\epsilon_{\infty}}=\sqrt{\frac{\pi}{4 i \omega \tau_{K}}} \exp \left(\frac{1}{4 i \omega \tau_{K}}\right) \operatorname{erfc}\left(\sqrt{\frac{1}{4 i \omega \tau_{K}}}\right) .
$$

This expression clearly cannot be represented exactly by any of the empirical functions in frequency domain.

Therefore, considerable effort has been made in the literature to establish approximate relationships between the Kohlrausch function and frequency domain expressions. Starting from the asymptotic properties of the Kohlrausch function that $\epsilon^{\prime \prime}(\omega) \sim \omega$ for low frequencies and $\epsilon^{\prime \prime}(\omega)$ $\sim \omega^{-\beta}$ for high frequencies the Cole-Davidson function with $\gamma=\beta$ seems to be the appropriate choice. But Fig. 3(b) shows that the high- and low-frequency wings have different levels and will not coincide despite having the same slope.

Therefore, it may be a better compromise for representing actual data to choose a mediatory value $\gamma<\beta$. Such an attempt was first described by Lindsey and Patterson (LP). ${ }^{18}$ On grounds of a least-squares fit they propose the relation

$$
\beta=\left\{\begin{array}{lc}
0.970 \gamma+0.144 & \text { for } 0.2 \leqslant \gamma \leqslant 0.6, \\
0.683 \gamma+0.316 & \text { for } 0.6 \leqslant \gamma .
\end{array}\right.
$$

Using the logarithmic moments derived here an alternative correspondence can be established by imposing that the moments up to the second should coincide. This results in the relation 

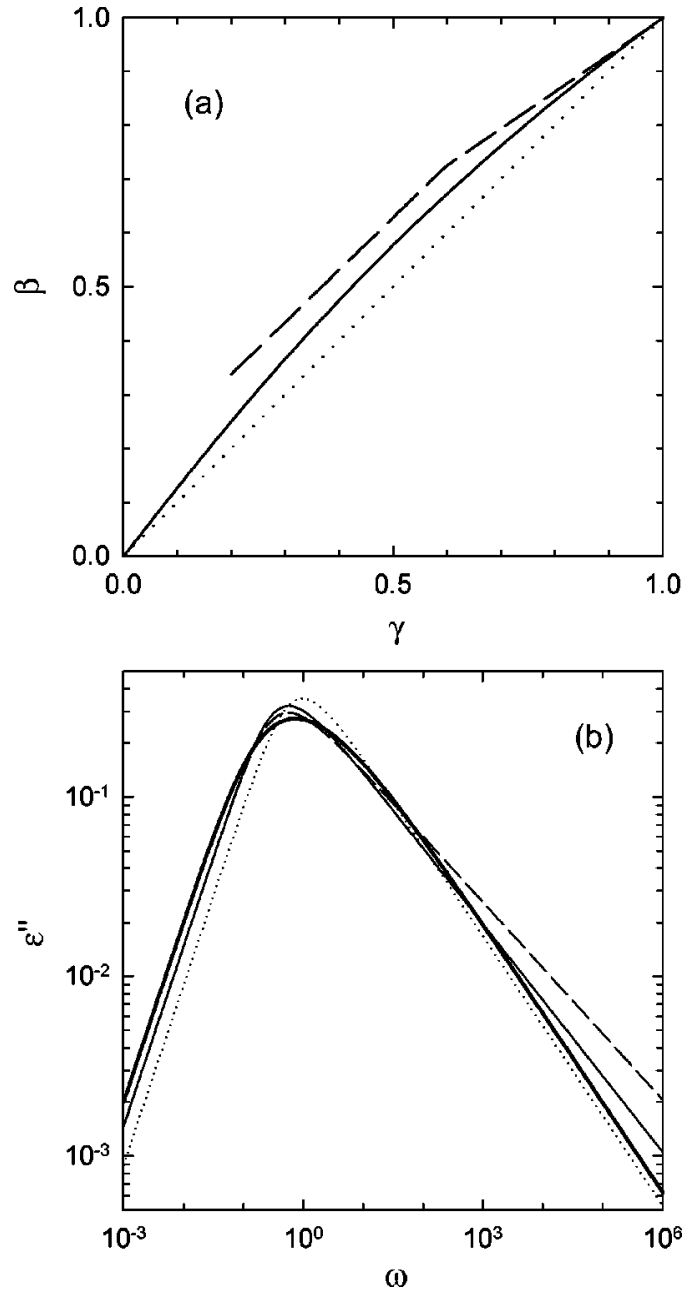

FIG. 3. (a) Correspondence between Cole-Davidson exponent $\gamma$ and Kohlrausch exponent $\beta$ resulting from equating the second logarithmic moment (continuous curve). For comparison the "least-squares" correspondence established by Lindsey and Patterson (Ref. 18) (dashed curve) and the correspondence from asymptotic behavior (dotted curve). (b) The performance of the three approximations demonstrated by comparing $\epsilon^{\prime \prime}(\omega)$ for $\beta=1 / 2$. The line styles for the Cole-Davidson curves are chosen as in (a), the Fourier transform of the Kohlrausch function (24) is plotted in bold.

$$
\beta=\frac{\pi}{\sqrt{6 \psi^{\prime}(\gamma)}} .
$$

Figure 3(a) shows a comparison of the correspondences. It can be seen that the one based on the logarithmic moments lies in between the LP formula and the "asymptotic" one. Concerning the agreement the LP correspondence performs slightly better in the peak while the logarithmic moments approach works better on the high frequency side. In both cases the low frequency wing is poorly represented.

In order to overcome this deficiency one can use a twoparameter function, namely Havriliak-Negami, as was first described by Alvarez, Alegría, and Colmenero (AAC). ${ }^{19}$ They use a numerical algorithm to invert (6) and thus obtain the distribution function of the Kohlrausch relaxation. Subsequently, they use this distribution to calculate $\epsilon_{K}^{\prime \prime}(\omega)$ from (7). Finally, the Havriliak-Negami function is determined with the parameters for which $\epsilon_{\mathrm{HN}}^{\prime \prime}(\omega)$ fits best to the previously calculated function. From the tabulated values of cor- responding exponent parameters, the following approximate relation to the Kohlrausch $\beta$ is established for given Havriliak-Negami parameters:

$$
\beta=(\alpha \gamma)^{0.813} \text {. }
$$

For a given Kohlrausch $\beta$, the additional relation

$$
\gamma=1-0.8121(1-\alpha)^{0.387}
$$

together with (27) fixes the optimal $\alpha, \gamma$ pair to be used. The characteristic times are related by

$$
\log _{10} \frac{\tau_{\mathrm{HN}}}{\tau_{K}}=2.6(1-\beta)^{0.5} \exp (-3 \beta) .
$$

The approach by logarithmic moments equating the second moments yields the following relation for the Kohlrausch $\beta$ with given Havriliak-Negami parameters:

$$
\beta=\left(\frac{1}{\alpha^{2}}+\frac{6 \psi^{\prime}(\gamma)}{\pi \alpha^{2}}-1\right)^{-1 / 2} \text {. }
$$

For given $\beta$ the corresponding Havriliak-Negami parameters can be obtained by this equation together with the following equation resulting from the third moments:

$$
2 \zeta(3)\left(1-\frac{1}{\beta^{3}}\right)=\frac{\psi^{\prime \prime}(\gamma)+2 \zeta(3)}{\alpha^{3}} .
$$

Finally, the ratio of the characteristic times is given by

$$
\ln \frac{\tau_{\mathrm{HN}}}{\tau_{K}}=\left(1-\frac{1}{\beta}-\frac{1}{\alpha}\right) \mathrm{Eu}-\frac{\psi(\gamma)}{\alpha} .
$$

Figure 4 shows the comparison of both parameter correspondence schemes. From Fig. 4(a) one can see that for Kohlrausch parameters $\beta \geqslant 0.3$ both methods yield the same Havriliak-Negami parameters within a \pm 0.03 range. Nevertheless, the conversion to $\beta$ of Havriliak-Negami parameters which are closer to the symmetric case $\gamma=1$ leads to larger differences. Interestingly, the discrepancy here is smallest for $\alpha=1$, i.e., the Cole-Davidson case.

The comparison of corresponding $\epsilon^{\prime \prime}(\omega)$ in Fig. 4(b) has a similar result as for the Cole-Davidson case before. The AAC correspondence (derived by a fit algorithm similar to LP) performs better close to the peak with clear deviations in the wings. Equating the logarithmic moments in contrast leads to a more "distributed" occurrence of the differences.

\section{CONCLUSIONS}

The use of logarithmic moments to characterize nonexponential relaxations can be justified by several arguments:

(1) Many dynamical experiments (especially light scattering $^{20}$ and dielectric spectroscopy ${ }^{21}$ ) can at present be done on enormous time or frequency scales. These can extend up to 18 decades. In such experiments data is necessarily taken on a logarithmic abscissa. Therefore, moments on that scale capture the information obtained better than those on a linear scale. For example, the average relaxation time $\langle\tau\rangle$ may be dominated by the low frequency part of the experiment while the average of the $\operatorname{logarithm}\langle\ln \tau\rangle$ has to take the whole spectrum into account. 

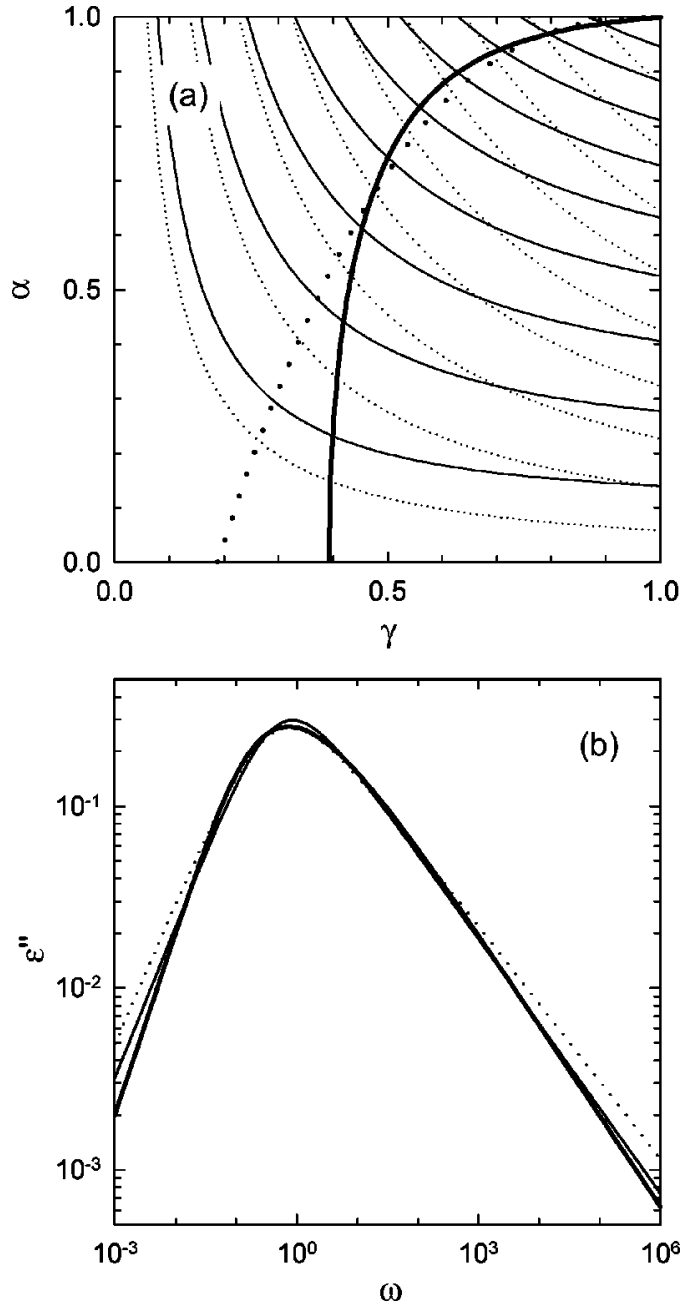

FIG. 4. (a) Correspondence between Havriliak-Negami exponents $\gamma, \alpha$ and Kohlrausch exponent $\beta$ resulting from equating the logarithmic moments (continuous curves). The thin lines show the $\gamma, \alpha$ pairs corresponding to constant $\beta=0-1$ in steps of 0.1 according to the second moment relation (30). The thick line represents the "optimal" $\gamma, \alpha$ pairs fulfilling also the third moment relation (31). The dotted curve shows for comparison the correspondence established by Alvarez, Alegría, and Colmenero (Ref. 19). (b) The performance of the two approximations demonstrated by comparing $\epsilon^{\prime \prime}(\omega)$ for $\beta=1 / 2$. The line styles for the Havriliak-Negami curves are chosen as in (a), the Fourier transform of the Kohlrausch function (24) is plotted in bold.

(2) A common way to interpret relaxation time distributions is to assume their origin in a distribution of energy barriers of an activated process. Such a distribution $G(E)$ via the Arrhenius law

$$
\tau=\tau_{0} \exp \left(\frac{E}{k_{B} T}\right)
$$

(with a unique prefactor $\tau_{0}$ ) leads to a distribution of relaxation times

$$
g(\ln \tau)=k_{B} T G\left(k_{B} T\left(\ln \tau-\ln \tau_{0}\right)\right) .
$$

In this picture the moments of the energy barrier distribution are related to the logarithmic moments of the relaxation time distribution in a simple way,

$$
\langle E\rangle=k_{B} T\left(\langle\ln \tau\rangle-\ln \tau_{0}\right),
$$

$$
\begin{aligned}
& \sigma_{E}=k_{B} T \sigma_{\ln \tau}, \\
& \gamma_{1 E}=\gamma_{1 \ln \tau} .
\end{aligned}
$$

Here, $\sigma_{E}^{2}$ and $\gamma_{1 E}$ are defined analogously by replacing $\ln \tau$ by $E$ in Eqs. (9)-(11). Apart from opening the possibility to calculate the moments of $G(E)$ without assuming a functional form and fitting complicated convolution expressions this can be seen as an a priori argument to characterize stretched relaxations by their logarithmic moments.

(3) The logarithmic moments can be directly calculated from experimental data. This allows the spectral information to be condensed into a set of numerical values without assuming a specific functional form as in the procedure of fitting free parameters. Expressions (14)-(16) can be used for frequency domain data, e.g., the dielectric loss $\epsilon^{\prime \prime}(\omega)$. Expressions (19)-(21) are appropriate for time-domain spectroscopy.

It has been shown that the logarithmic moments can be calculated in closed form for all empirical fit formulas which are currently in use. This allows the use of literature data also in cases where "raw" spectra are not presented. By comparing the logarithmic moments results from different sources can be compared also if the authors prefer different fit schemes (e.g., Havriliak-Negami and Jonscher).

Finally, logarithmic moments can be used to derive approximate relations between time domain functions and frequency spectra where an exact Fourier transform is impossible (as, e.g., for the Kohlrausch expression). These seem to be valid over a larger dynamical range than those derived by least-squares methods.

${ }^{1}$ R. Kohlrausch, Ann. Phys. (Leipzig) 91, 56 (1854).

${ }^{2}$ K. S. Cole and R. H. Cole, J. Chem. Phys. 9, 341 (1941).

${ }^{3}$ D. W. Davidson and R. H. Cole, J. Chem. Phys. 18, 1417 (1950); 19, 1484 (1951).

${ }^{4}$ S. Havriliak and S. Negami, J. Polym. Sci., Part C: Polym. Symp. 14, 99 (1966).

${ }^{5}$ For reasons of conformity the exponent parameters are defined here in a way that their value 1 always corresponds to the Debye case. With respect to the original publications this means a substitution $1-\alpha \rightarrow \alpha$ (Cole-Cole and Havriliak-Negami) and $m \rightarrow a, 1-n \rightarrow b$ (Jonscher).

${ }^{6}$ R. M. Fuoss and J. G. Kirkwood, J. Am. Chem. Soc. 63, 385 (1941).

${ }^{7}$ A. K. Jonscher, Colloid Polym. Sci. 253, 231 (1975).

${ }^{8} \mathrm{~W}$. Feller, An Introduction to Probability Theory and Its Applications (Wiley, New York, 1971).

${ }^{9}$ S. W. Provencher, Comput. Phys. Commun. 27, 213 (1982).

${ }^{10}$ F. D. Morgan and D. P. Lesmes, J. Chem. Phys. 100, 671 (1994).

${ }^{11}$ J. G. Kirkwood and R. M. Fuoss, J. Chem. Phys. 9, 329 (1941).

${ }^{12}$ H. Fröhlich, Theory of Dielectrics (Clarendon, Oxford, 1949), p. 93.

${ }^{13}$ A. Matsumoto and K. Higasi, J. Chem. Phys. 36, 1776 (1962).

${ }^{14}$ A. K. Rajagopal and K. L. Ngai, in Relaxations in Complex Systems, edited by K. L. Ngai and G. B. Wright (National Technical Information Service, Springfield, 1984), p. 275.

${ }^{15}$ C. J. F. Böttcher and P. Bordewijk, Theory of Electric Polarization (Elsevier, Amsterdam, 1978), Vol. II.

${ }^{16}$ S. Havriliak and S. J. Havriliak, J. Non-Cryst. Solids 172-174, 297 (1994).

${ }^{17}$ The Fourier integral is a special case of formula 2.3.2.13 or 2.5.2.11 in A. P. Prudnikov, Yu. A. Brychkov, and O. I. Marichev, Integrals and Series (Gordon and Breach, New York, 1992).

${ }^{18}$ C. P. Lindsey and G. D. Patterson, J. Chem. Phys. 73, 3348 (1980).

${ }^{19}$ F. Alvarez, A. Alegría, and J. Colmenero, Phys. Rev. B 44, 7306 (1991).

${ }^{20}$ For example, W. Steffen, A. Patkowski, H. Gläser, G. Meier, and E. W. Fischer, Phys. Rev. E 49, 2992 (1994).

${ }^{21}$ For example, U. Schneider, P. Lunkenheimer, R. Brand, and A. Loidl, J. Non-Cryst. Solids 235-237, 173 (1998). 